\title{
Head and neck sarcomas: A single institute series.
}

Leandros-Vassilios Vassiliou DDS, MD, MRCS, MSc ${ }^{1}$, Bagrat Lalabekyan DDS, MBBS, $\mathrm{PhD}^{1}$, Amrita Jay BDS, MDS, MFDSRCS, FRCPath ${ }^{1}$, Colin Liew FRCS ${ }^{1}$, Jeremy Whelan MD, FRCP, MBBS ${ }^{1}$, Laurence Newman FRCS, LLM ${ }^{2}$, Nicholas Kalavrezos FRCS, FFD, $\mathrm{MD}^{1}$.

${ }^{1}$ University College London Hospital (UCLH), London, UK

${ }^{2}$ The Queen Victoria Hospital, East Grinstead, UK

Corresponding author:

Mr Leandros Vassiliou, Department of head and neck, University College London Hospital (UCLH), 250 Euston Road, NW12PG, London, UK.

Telephone: +44 (0) 7595308387

Fax: $+44(0) 2034479855$

Word Count: 2878 
Introduction

Sarcomas are malignant neoplasms of mesenchymal origin that comprise less than $1 \%$ of all cancers. They demonstrate aggressive biological behaviour, with the majority being locally invasive with significant potential for metastasis. ${ }^{1,2}$ Sarcomas are generally divided into bone sarcomas (BS) and soft tissue sarcomas (STS).

The overall annual incidence of BSs is $8 / 10^{6}$. On average 38 BSs of the skull and facial skeleton are diagnosed in England annually. These account for $10 \%$ of all BS. ${ }^{1,3}$ There is a male predilection and a bimodal age-specific distribution (second and third decade). Osteosarcomas, Ewing sarcoma and chondrosarcoma are the main histological subtypes. ${ }^{1}$

The overall annual incidence of STSs is $30 / 10^{6}$, with slight male predominance and the estimated median age at presentation is 65 years. ${ }^{1,4,5}$ On average 190 STSs of the head and neck region are diagnosed annually in England, accounting for $9 \%$ of all soft tissue sarcomas. ${ }^{4}$ They are histologically diverse with more than 50 described subtypes. ${ }^{1}$

Of all adult sarcomas, only 5-15\% occur in the head and neck region, with 5-year survival rates ranging from $27 \%$ to $84 \%$ in various studies. ${ }^{6-10}$

The mainstay of treatment for BS is radical surgery, preceded by neo-adjuvant chemotherapy for high grade tumours. This treatment model is an extrapolation of the management applied to long bone sarcomas especially in limb-sparing surgery. ${ }^{11}$ The aim of neo-adjuvant chemotherapy in BS of the head and neck is two-fold: elimination of distant -lung predominantly- metastases and improvement of local control by reducing the need for large uninvolved soft tissue excision margins at the primary tumour site, which in many cases is difficult due to the complex anatomy of the head and neck region. The role of radiotherapy in head and neck BS is limited, with the exception of Ewing sarcomas. However, radiotherapy 
is appropriate for the management of residual disease in cases of positive resection margins, when surgical re-excision is not feasible, or when the lesion recurs in anatomically inaccessible areas. $^{11}$

Treatment for head and neck STS varies, depending on the specific histopathological type, grade and extent of the tumour. The threshold for using neo-adjuvant chemotherapy may be lower than in soft tissue sarcomas of the extremities, given the challenges of achieving local control. Radical radiotherapy is appropriate for similar indications as for BS. Surgery is implemented in the management of STS in order to maximise the chances of disease control. The role of surgery in STS depends on the resectability of the disease. Its timing -prior or after radiotherapy- depends mainly upon the specific reconstructive aims and the healing potential of radiotherapy-treated tissues. The management strategy is planned and reviewed within a specific sarcoma multidisciplinary setting with radiological, histopathological, oncological and surgical expertise.

This paper reports a cohort of patients with head and neck sarcomas managed by the same surgical and medical team over a period of 13 years. We describe our experience and the evolution of surgical concepts adapted to the histopathological patterns and biological behaviour of these distinct groups of non-epithelial head and neck malignancies. 


\section{Materials \& Methods}

This study is in line with our institutional governance protocol. We analysed the records of head and neck oncology patients treated between 1997-2010. In this 13 years period the three senior authors (NK, LN and JW) consistently managed all head and neck sarcoma patients.

High grade BS were routinely considered for neo-adjuvant chemotherapy using standard regimens in the absence of significant co-morbidities. In most cases the goal was to complete chemotherapy prior to the definitive surgical resection with the response assessment undertaken using PET-CT. ${ }^{12}$ For patients with Ewing sarcomas, the use of radiotherapy instead of surgery was considered on the basis of individual tumour characteristics and response. Patients with chondrosarcomas were treated surgically. Patients with STS underwent primary resection followed by post-operative radiotherapy depending on resection histology.

All statistical analysis was performed using the statistical software package SPSS 12.0 (IBM, New York, USA). Survival estimates (Overall Survival and Event-free survival) have been calculated using the Kaplan-Meier method. ${ }^{13}$ Complete follow-up data was available for 101 patients. The follow-up was calculated in months. For the event-free survival estimates, recurrence, metastasis or disease-specific death have been classified as 'event'. The log-rank test was used for uni-variate survival comparison. ${ }^{14}$ In chi-square analysis $p$ values $<0.1$ were considered significant. 
Results

A total of 107 patients with head and neck sarcomas were identified. The histopathological subtypes of bone and soft tissue sarcomas are illustrated in Table 1.

\section{Bone Sarcomas}

Fifty-four patients had BS (33 males, 21 females, male/female ratio: 1.6:1). The mean age was 41 years (range 9-81 years). Fifty cases were primary localised bone sarcomas, whereas two were recurrent from previously elsewhere treated tumours, one was already metastatic at presentation and the fourth was a secondary deposit from a non head-neck sarcoma (these cases were not included in the subsequent survival analysis).

Bone sarcomas predominantly arose in the mandible $(29 / 54,54 \%)$, followed by the maxilla $(19 / 54,34 \%)$, whereas six cases occurred in extragnathic locations $(6 / 54,11 \%)$, namely one in the frontal bone, one in the sphenoid bone, one sinonasal and three chondrosarcomas arising from the laryngeal structures. The majority of the tumours (49 out of 54, 91\%) were high grade. Eight osteosarcomas were radiation induced.

Neoadjuvant chemotherapy was used in 28 osteosarcomas and in the 5 cases of Ewing's sarcomas followed by surgery (Figure 1). Twenty-five mandibulectomies and 21 maxillectomies were performed, with the type and extent of resections is presented in Table $2 .^{15,16}$

Four cases comprised en bloc resections of both hemi-mandible and maxilla in the context of compartmental excision along with lateral access and base of skull clearance.

In osteosarcomas when histopathology reported involved resection margins, re-excision was performed. Out of 39 surgically treated osteosarcomas where histological data were retrieved, 
35 were completely excised. Three mandibular osteosarcoma cases with tumour dimensions above $8 \mathrm{~cm}$ were extending into the cranial base were the excision margins were reported involved. The fourth case was a radiation induced sarcoma, with disease extending along the totality of the mandible and a substantial part of the floor of the mouth.

A significant observation was noted early on bone sarcomas centred at the retromolar region extending proximally to the vertical ramus of the mandible. Although Magnetic Resonance Imaging (MRI) is considered to be the gold standard in assessing bone marrow invasion, in two cases the extent of the disease in the condylar area was grossly underestimated. In those cases the histopathology analysis demonstrated florid disease infiltration of the condylar head without exceeding the end plate of the cortex causing no cortical alterations and therefore no visible imaging changes (Figure 2). It is our policy now to perform hemi-mandibulectomy with condylar disarticulation in osteosarcomas that extend into the mandibular ramus. We have performed 12 condylar disarticulation resections, that were subsequently ratified by the histological analysis.

The majority of mandibulectomy defects were reconstructed with vascularized composite flaps (15 fibulas, 4 DCIA flaps). In three cases no bone reconstruction was attempted due to substantial co-morbidities and the composite defect was closed using myocutaneous pedicled latissimus dorsi flaps. One patient aged 9 years was reconstructed with a costochondral graft. The maxillectomy defects were reconstructed primarily with free flaps (three fibulas and one DCIA), the rest been obturated. For bi-maxillary defects one chimeric scapula, one DCIA and two large composite fibula flaps were used.

Radiotherapy (IMRT) was delivered in 10 cases; in four for positive margins in unresectable areas and in the rest for palliation.

Eight low grade chondrosarcomas were operated. In three laryngeal cases with slow growth radical surgery was avoided and tumours were incompletely excised. 
Follow-up was recorded for 51 bone sarcoma patients (mean 48 months).

The 2- and 5-year overall survival (OS) estimates were 91\% and 73\% (data available for $n=51$ ). The 2- and 5-year event-free survival rates for BSs were $66 \%$ and $49 \%$ (Figure 3 ).

\section{Soft tissue sarcomas}

Fifty-three patients had STSs (28 male, 25 female, male/female ratio: 1.1:1). Mean age was 43 years (range 7-86 years). Six patients with soft tissue sarcomas had already developed metastatic disease at the time of their presentation. Information about tumour grade was not available for 19 tumours. Out of 34 tumours where grade data was available, 26 (76\%) were high grade and $8(24 \%)$ were low grade. Six tumours were radiation induced. Five patients had distant metastatic disease at the time of the diagnosis. Two of these cases were rhabdomyosarcomas, one myxofibrosarcoma, one malignant peripheral nerve sheath tumour and one angiosarcoma.

The site distribution of soft tissue sarcomas was widely dispersed. We utilised a site-specific classification based on the anatomical landmarks set by Roon, Christensen ${ }^{17}$ and Ohngren ${ }^{18}$ to divide the head and neck region in four zones (Figure 4):

I) High - above the angle of the mandible. This area is further divided by Ohngren's line (medial canthus to angle of mandible) into the region Ia antero-inferiorly, which encompasses the facial anatomical boundary, and $\mathrm{Ib}$ postero-superiorly, which contains the skull base, infratemporal, pterygopalatine and retrobulbar spaces.

II) Middle - between the angle of the mandible and the lower border of the cricoid cartilage.

III) Low - below the cricoid cartilage. 
The distribution pattern of the tumours' epicentres is illustrated in Figure 4.

Neoadjuvant chemotherapy followed by surgery was used in 20 out of 53 STSs, mainly for rhabdomyosarcomas and high grade sarcomas for which resection was anticipated to result in marginal or involved margins. Surgery was employed in 39 cases .

The tumours were resected by wide compartmental excision. For tumours sited in zone I, 9 mandibulectomies and 6 maxillectomies were performed, including 3 cases were en bloc mandibulo-maxillary resections along with skull base clearance were performed (Table 2). Sixteen free flap reconstructions were performed.

Clear surgical margins were achieved in 25/39 (64\%) of the cases (Figure 4). Clearance was achieved in $72 \%$ of zone Ia, $33 \%$ of zone $\mathrm{Ib}(59 \%$ in Zone I in total), $80 \%$ of zone II and $50 \%$ of zone III tumours. The difference in tumour clearance between zone Ia and $\mathrm{Ib}$ was significant. In zone III $3 / 5$ of tumours were unresectable due to extension into the thoracic cavity with subsequent invasion of vital structures.

Radiotherapy was delivered in a total of 29 cases. In 11 out of 43 operated cases radiotherapy was administered for positive margins in unresectable disease.

The 2- and 5-year overall survival rates were $67 \%$ and $56 \%$ respectively (data available for $\mathrm{n}=50$ ) (Figure 5). The 2- and 5-year event-free survival rates were 54\% and 47\%, respectively.

The survival rates of all the cases divided by histological type are demonstrated on Table 3 . 


\section{Discussion}

Sarcomas are a widely heterogeneous group of malignant tumours. Their rarity in the head and neck region accounts for the lack of consistency in the literature regarding their management and outcomes. There are no randomised controlled studies and the existing metanalyses provide controversial results. ${ }^{19,20}$

In this study, we report the outcomes of a large BS and STS series. Mean age at presentation and site distribution were consistent with those published in the literature. ${ }^{21,}{ }^{22}$ Soft tissue sarcomas series are largely variable due to the rarity, diversity and heterogeneity of these tumours. $^{23}$

In our series, the majority of bone $(91 \%)$ and soft tissue $(76 \%)$ sarcomas were high-grade. The incidence of high-grade tumours in the head and neck region is reported to vary between $56 \%$ and $79 \%$ in osteosarcomas. ${ }^{24,25}$

Surgery for sarcomas is based on the principles of oncological ablative and reconstructive surgery, however distinct factors related to the disease characteristics and the usage of neoadjuvant chemotherapy dictate additional variables in the concept of their excision. The mesenchymal origin of these tumours in contrast with the majority of the head and neck epithelial-derived cancers, predisposes to a different pattern of disease spread and potentially different extent of cancellous bone infiltration (Supplementary data).

The importance of surgical resection of BSs with clear margins has been emphasized in the paper of Chen et al. ${ }^{26}$ Regarding the target resection margin, some groups advocate a $3 \mathrm{~cm}$ macroscopic margin. ${ }^{8}$ In conventional trunk and extremities osteosarcomas, most studies suggest that a $2 \mathrm{~cm}$ macroscopic margin is adequate. ${ }^{27,}{ }^{28}$ This has been reinforced by the input of neoadjuvant chemotherapy, which potentially eliminates microsatellite tumour 
deposits that extend beyond the primary tumour. ${ }^{29,}{ }^{30}$ An important key-point is, that where neoadjuvant treatment (chemotherapy) has been employed, the resection should be planned based on the pre-treatment scans. Surgical planning based solely on post-neo-adjuvant treatment imaging, especially in cases with radiologic good response, may be misleading due to the frequently encountered partial and certainly non-concentric pattern of tumour "shrinkage".

A simplistic geometrical approach of 'measuring' centimetres around the tumour in the anatomically complex head and neck region is not applicable. Our experience has shown that sufficient margins are only achieved when performing a wide 'compartmental' excision, aiming at large bony margins with concomitant complete excision of the surrounding soft tissue envelope. The removal of the involved anatomical compartment has to be performed in a fashion wide enough to encompass all potentially involved structures taking into account the potential of bone marrow involvement beyond the radiological reported 'margins' (Figure 2). To this end frank hemi-mandibulectomy and even condylar disarticulation have to be considered when the tumour, especially a high-grade osteosarcoma, extends to the mandibular ramus. Preservation of a small condylar segment may have little to offer in the subsequent reconstruction but most importantly can inhibit access to the base of skull when this is needed (i.e. infratemporal fossa involvement) jeopardising soft tissue clearance. In our experience mandibular reconstruction with composite fibula facilitates satisfactory mouth opening, while -not uncommonly- we have noticed that preservation of a small condylar segment may result in "late" anterior-medial displacement due to lateral pterygoid muscle traction. In this reported series of osteosarcomas, surgical clearance was achieved in $85 \%$ of the cases.

In soft tissue sarcomas the aim is a macroscopic margin of $1-3 \mathrm{~cm}$ where possible. ${ }^{31}$ The importance of the marginal status in the disease free and overall survival has been clearly 
demonstrated. ${ }^{9,23,31}$ It is widely accepted that surgical clearance in STSs is more difficult to achieve. $^{9,} 10,23,32$ Reasons are the biological aggressiveness of soft tissue sarcomas and the pattern of infiltration into the neighbouring vital neurovascular structures limiting their amenability for radical resection. Surgical clearance in our series of soft tissue sarcomas was achieved in 25/39 (64\%) of our cases. The experience gained from this series suggests that tumours arising within zone $\mathrm{Ib}$ or large tumours form adjacent areas extending into it are particularly difficult to clear. Eleven out of 14 incompletely excised head and neck soft tissue sarcomas were in zone I ( 6 arising within zone $\mathrm{Ib}$ and 5 extending from Ia into zone $\mathrm{Ib}$ ). This site-specific classification especially for soft tissue sarcomas can serve as a potential prognosticator aiding the decision-making algorithm when initially planning the management of these patients. In tumours confined in zone Ia surgery plays a pivotal role, whereas in zone Ib tumours poor surgical outcomes may be encountered.

In this series the overall survival estimate for BSs $(n=51)$ reached $91 \%$ at 2 years and $73 \%$ at 5 years, whilst for STSs $(\mathrm{n}=50) 67 \%$ at 2 years and $56 \%$ at 5 years, respectively. Corresponding studies report overall survival rates of $84 \%$ at 2 years and $61 \%$ at 5 years for mixed bone and soft tissue tumours ${ }^{10}, 79 \%$ at 2 years and $74 \%$ at 5 years ${ }^{22}$ and $63 \%$ at 5 years $^{21}$ for BSs, and $57 \%$ at 5 years for STSs $(n=50) .{ }^{33}$ In our series the bone sarcomas displayed significantly better overall survival probability ( $\mathrm{p}=0.0029$, Log-rank test) comparing to soft tissue sarcomas.

Although the outcomes of this series are comparable to the majority published in the literature, we believe that comparisons of survival estimates among different studies are difficult, due to the heterogeneity of histopathological types, the variations in the composition of studied populations and the diversities in the follow-up time spans. For example, the survival outcomes reported in our series, are the cumulative ones for all the patients managed in our centre, including cases with secondary or metastatic tumours. Interestingly, in our 
cohort we included 14 cases of radiation-induced tumours. Radiation-induced sarcomas are associated with significantly poorer outcome than that of the stage-matched irradiationindependent counterpart sarcomas, with overall 5-year survival rates as low as $25 \%$. The attributing factors include delay in diagnosis, biological aggressiveness, chemotherapy resistance and the limitation in treatment options, especially the ineligibility for radiotherapy and the technical difficulties in obtaining clear margins because of frequent proximity of the tumours to major neurovascular structures. ${ }^{34-36}$

As a retrospective analysis, the current study is subjected to limitations. In this series the wide histopathological diversity, especially with the soft tissue sarcomas comprising a conglomerate of heterogeneous subtypes and anatomical locations, did not allow stratified multi-variate analysis.

A meta-analysis including all series published by different centres, or even randomised clinical trials will be useful to address fundamental questions in the field of head and neck sarcomas management.

\section{Conclusions}

Surgery has to consider the degree of bone marrow infiltration and aim for a wide 'compartmental' excision with large bony margins and sufficient surgical clearance of the soft tissue envelope in the surgical treatment of bone sarcomas. Soft tissue sarcomas are histologically extremely diverse and surgery should be the primary modality of treatment in relatively small disease burden or cases of low/ moderate grading. 
References

1. Fletcher Christopher D.M., Unni K.Krishnan, Mertens Fredrik. Pathology and Genetics of Tumours of Soft Tissue and Bone. In: World Health Organization Classification of Tumours. IARC WHO Classification of Tumours No 5, IARC Press $5 ; 2002$.

2. Burningham Z, Hashibe M, Spector L, Schiffman JD. The epidemiology of sarcoma. Clin Sarcoma Res 2012;2(1):14.

3. Whelan J, McTiernan A, Cooper $\mathrm{N}$ et al. Incidence and survival of malignant bone sarcomas in England 1979-2007. Int J Cancer 2012;131(4):E508-E517.

4. http://www.cancerresearchuk.org/cancer-info/cancerstats/keyfacts/soft-tissuesarcoma/.2015.

5. http://www.ncin.org.uk/cancer_type_and_topic_specific_work/cancer_type_specific_ work/sarcomas/.2015.

6. Oda D, Bavisotto LM, Schmidt RA et al. Head and neck osteosarcoma at the University of Washington. 1997;19:513-523.

7. August M, Magennis P, Dewitt D. Osteogenic sarcoma of the jaws: Factors influencing prognosis. International Journal of Oral and Maxillofacial Surgery 1997;26(3):198-204.

8. Fernandes R, Kolokythas A, Pazoki A, Nikitakis NG, Ord RA. Sarcomas of the head and neck: a 10 year experience with 37 patients at the University of Maryland. Oral Oncology 2005;1(1):131-132.

9. Kraus DH, Dubner S, Harrison LB et al. Prognostic factors for recurrence and survival in head and neck soft-tissue sarcomas. 1994;74:697-702.

10. Mucke T, Mitchell DA, Tannapfel A et al. Outcome in Adult Patients With Head and Neck Sarcomas-A 10-Year Analysis. Journal of Surgical Oncology 2010;102(2):170174.

11. Grimer R, Athanasou N, Gerrand C et al. UK Guidelines for the Management of Bone Sarcomas. Sarcoma 2010;2010:317462. 
12. Frezza AM, Beale T, Bomanji $\mathrm{J}$ et al. Is [F-18]-fluorodeoxy-D-glucose positron emission tomography of value in the management of patients with craniofacial bone sarcomas undergoing neo-adjuvant treatment? BMC Cancer 2014;14:23.

13. Kaplan EL, Meier P. Nonparametric-Estimation from Incomplete Observations. Journal of the American Statistical Association 1958;53(282):457-481.

14. Peto R, Pike MC, Armitage P et al. Design and Analysis of Randomized ClinicalTrials Requiring Prolonged Observation of Each Patient .2. Analysis and Examples. British Journal of Cancer 1977;35(1):1-39.

15. Boyd JB, Gullane PJ, Rotstein LE, Brown DH, Irish JC. Classification of mandibular defects. Plast Reconstr Surg 1993;92(7):1266-1275.

16. Spiro RH, Strong EW, Shah JP. Maxillectomy and its classification. Head Neck 1997;19(4):309-314.

17. Roon AJ, Christensen N. Evaluation and treatment of penetrating cervical injuries. J Trauma 1979;19(6):391-397.

18. Ohngren LG. Malignant tumors of the maxillo-ethmoidal region. Acta Otolaryngologica 1933;Supplement 19:101-106.

19. Kassir RR, Rassekh CH, Kinsella JB, Segas J, Carrau RL, Hokanson JA. Osteosarcoma of the head and neck: Meta-analysis of nonrandomized studies. 1997;107:56-61.

20. Smeele LE, Kostense PJ, vanderWaal I, Snow GB. Effect of chemotherapy on survival of craniofacial osteosarcoma: A systematic review of 201 patients. $1997 ; 15: 363-367$.

21. Guadagnolo BA, Zagars GK, Raymond K, Benjamin RS, Sturgis EM. Osteosarcoma of the Jaw/Craniofacial Region Outcomes After Multimodality Treatment. Cancer 2009;115(14):3262-3270.

22. Jasnau S, Meyer U, Potratz J et al. Craniofacial osteosarcoma - Experience of the cooperative German-Austrian-Swiss osteosarcoma study group. Oral Oncology 2008;44(3):286-294.

23. Patel SG, Shaha AR, Shah JP. Soft tissue sarcomas of the head and neck: An update. 2001;22:2-18. 
24. Mardinger O, Givol N, Talmi YP, Taicher S, Saba K, Hashomer T. Osteosarcoma of the jaw - The Chaim Sheba Medical Center experience. Oral Surgery Oral Medicine Oral Pathology Oral Radiology and Endodontics 2001;91(4):445-451.

25. Clark JL, Unni KK, Dahlin DC, Devine KD. Osteo-Sarcoma of the Jaw. Cancer 1983;51(12):2311-2316.

26. Chen Y, Shen Q, Gokavarapu S et al. Osteosarcoma of head and neck: A retrospective study on prognostic factors from a single institute database. Oral Oncol 2016;58:1-7.

27. Enneking WF, Spanier SS, Goodman MA. A system for the surgical staging of musculoskeletal sarcoma. 1980;106-120.

28. Kawaguchi N, Ahmed AR, Matsumoto S, Manabe J, Matsushita Y. The concept of curative margin in surgery for bone and soft tissue sarcoma. 2004;165-172.

29. Picci P, Sangiorgi L, Rougraff BT, Neff JR, Casadei R, Campanacci M. Relationship of chemotherapy-induced necrosis and surgical margins to local recurrence in osteosarcoma. 1994;12:2699-2705.

30. Li X, Moretti VM, Ashana AO, Lackman RD. Impact of close surgical margin on local recurrence and survival in osteosarcoma. International Orthopaedics 2012;36(1):131-137.

31. Zagars GK. Surgical margins and reresection in the management of patients with soft tissue sarcoma using conservative surgery and radiation therapy. 2003.

32. Wanebo HJ, Koness RJ, Macfarlane JK et al. Head and neck sarcoma - report of the head and neck sarcoma registry. 1992;14:1-7.

33. Van Damme JP, Schmitz S, Machiels JP et al. Prognostic factors and assessment of staging systems for head and neck soft tissue sarcomas in adults. Ejso 2010;36(7):684-690.

34. Cahan WG, Woodard HQ, Higinbotham NL, Stewart FW, Coley BL. Sarcoma arising in irradiated bone - Report of eleven cases. 1998;82:8-34.

35. Gutierrez VF, Pendas JLL, Pelaz AC, Farpon RC, Nieto CS. Radiation-induced sarcomas of the head and neck. 2008;19:1287-1291.

36. Huber GF, Matthews TW, Dort JC. Radiation-induced soft tissue sarcomas of the head and neck. Journal of Otolaryngology 2007;36(2):93-97. 
Figure legends

Figure 1. MRI scan of mandibular osteosarcoma. a. Prior to neoadjuvant chemotherapy; i. Coronal slice ii. Axial view. b. Post-6 cycles of MAP chemotherapy; i. Coronal slice. ii. Axial view.

Figure 2. Discrepancy between extent of tumour marrow invasion as seen in: a. histological specimen of the condylar head. b. MRI coronal sections of the same region 2 weeks preoperatively.

Figure 3. Kaplan-Meier survival estimates for bone sarcomas $(n=51)$. a. Overall survival. $b$. Event free survival estimates.

Figure 4. Site-specific classification for soft tissue sarcomas in relation to tumour clearance. Green: completely excised tumours. Red: Tumours excised with one or more positive margins. Blue: Non-operated tumours.

Figure 5. Kaplan-Meier survival estimates for soft tissue sarcomas $(n=50)$. a. Overall survival. b. Event free survival estimates.

Figure 6. Schematic presentation of tumour invasion pathway in the mandible. a. Epithelial tumour arising from mucosa infiltrating through the cortex into the medullary space $($ Out $\rightarrow$ In). b. Osteosarcoma arising from the medullary space infiltrating the bone marrow and penetrating through the bone into the soft tissues ( $\mathrm{In} \rightarrow \mathrm{Out}$ ). 
Table 1. Head and neck sarcoma cases breakdown. Histopathological classification and treatment modalities. *MPNST: malignant peripheral nerve sheath tumour

\begin{tabular}{|lcccc|}
\hline \multicolumn{1}{|c}{ HEAD \& NECK SARCOMAS } & $\begin{array}{c}\text { NUMBER } \\
\text { OF }\end{array}$ & SURGERY & $\begin{array}{c}\text { NEOADJUVANT } \\
\text { CHEMOTHERAPY }\end{array}$ & RADIOTHERAPY \\
\hline BONE SARCOMAS & 54 & $49 / 53$ & $33 / 53$ & $10 / 53$ \\
Osteosarcomas & 41 & $37 / 41$ & $28 / 41$ & $5 / 41$ \\
Ewing's sarcomas & 5 & $4 / 5$ & $5 / 5$ & $3 / 5$ \\
Chondrosarcomas & 8 & $8 / 8$ & $0 / 8$ & $2 / 8$ \\
& & & & $29 / 53$ \\
SOFT TISSUE SARCOMAS & 53 & $43 / 53$ & $20 / 53$ & $6 / 12$ \\
Rhabdomyosarcoma & 12 & $8 / 12$ & $4 / 12$ & $2 / 6$ \\
Spindle cell sarcoma & 6 & $6 / 6$ & $1 / 5$ & $4 / 5$ \\
Myxofibrosarcoma & 5 & $3 / 5$ & $1 / 3$ & $3 / 3$ \\
Synovial sarcoma & 3 & $3 / 3$ & $0 / 4$ & $2 / 4$ \\
Neurofibrosarcoma (MPNST $*$ & 4 & $4 / 4$ & $0 / 3$ & $0 / 3$ \\
Myofibrosarcoma & 3 & $2 / 3$ & $2 / 5$ & $4 / 5$ \\
Leiomyosarcoma & 5 & $4 / 5$ & $0 / 2$ & $1 / 2$ \\
Liposarcoma & 2 & $2 / 2$ & $1 / 2$ & $0 / 2$ \\
Dermatofibrosarcoma & 2 & $2 / 2$ & $0 / 1$ & $1 / 1$ \\
Angiosarcoma & 1 & $0 / 1$ & $1 / 1$ & $1 / 1$ \\
Alveolar soft part & 1 & $1 / 1$ & $3 / 9$ & $5 / 9$ \\
Undifferentiated/Pleomorphic & 9 & $8 / 9$ & & \\
\hline
\end{tabular}


Table 2. Extent of mandibulectomies and maxillectomies.

\begin{tabular}{|c|c|c|c|}
\hline \multicolumn{2}{|r|}{ MANDIBULECTOMIES } & \multicolumn{2}{|c|}{ MAXILLECTOMIES } \\
\hline \multicolumn{4}{|c|}{ Bone Sarcomas } \\
\hline \multicolumn{2}{|r|}{$N=25$} & \multicolumn{2}{|c|}{$N=21$} \\
\hline HLCL & 3 & Limited & 11 \\
\hline HLC & 3 & Subtotal & 8 \\
\hline HL & 8 & Total & 2 \\
\hline LC & 6 & Radical (incl. orbit) & 0 \\
\hline $\mathbf{L}$ & 4 & & \\
\hline C & 1 & & \\
\hline \multicolumn{4}{|c|}{ Soft tissue sarcomas } \\
\hline \multicolumn{2}{|r|}{$N=9$} & \multicolumn{2}{|c|}{$N=7$} \\
\hline HL & 4 & Limited & 0 \\
\hline LCL & 1 & Subtotal & 3 \\
\hline LC & 1 & Total & 2 \\
\hline $\mathbf{L}$ & 3 & Radical (incl. orbit) & 2 \\
\hline
\end{tabular}


Table 3. Survival estimates of Head \& Neck sarcomas based on the histopathological type.

\begin{tabular}{|c|c|c|c|c|c|c|c|c|c|}
\hline Histology & $\mathbf{N}$ & $\begin{array}{l}\text { 2-year } \\
\text { Overall } \\
\text { Survival }\end{array}$ & $\begin{array}{l}\text { Standard } \\
\text { Error (SE) }\end{array}$ & $\begin{array}{l}\text { 5-year } \\
\text { Overall } \\
\text { Survival }\end{array}$ & $\begin{array}{l}\text { Standard } \\
\text { Error (SE) }\end{array}$ & $\begin{array}{l}\text { 2-year } \\
\text { Event-Free } \\
\text { Survival }\end{array}$ & $\begin{array}{l}\text { Standard } \\
\text { Error (SE) }\end{array}$ & $\begin{array}{l}\text { 5-year } \\
\text { Event-Free } \\
\text { Survival }\end{array}$ & $\begin{array}{l}\text { Standard } \\
\text { Error (SE) }\end{array}$ \\
\hline \multicolumn{10}{|l|}{ Bone Sarcomas } \\
\hline Osteosarcomas (LG) & 4 & 0.75 & 0.22 & 0.75 & 0.22 & 0.75 & 0.217 & 0.75 & 0.217 \\
\hline Osteosarcomas (HG) & 26 & 0.95 & 0.046 & 0.74 & 0.11 & 0.783 & 0.88 & 0.595 & 0.116 \\
\hline Ewing's sarcomas & 5 & 0.8 & 0.179 & 0.8 & 0.179 & 0.8 & 0.179 & 0.8 & 0.179 \\
\hline Chondrosarcomas & 8 & 1.0 & . & 1.0 & . & 0.75 & 0.153 & 0.225 & 0.185 \\
\hline \multicolumn{10}{|l|}{ Soft Tissue Sarcomas } \\
\hline Rhabdomyosarcomas & 11 & 0.53 & 0.155 & 0.265 & 0.203 & 0.545 & 0.150 & 0.364 & 0.179 \\
\hline Myxofibrosarcomas & 5 & 0.8 & 0.179 & 0.53 & 0.248 & 0.8 & 0.179 & 0.533 & 0.248 \\
\hline Pleomorphic sarcomas & 5 & 0.8 & 0.179 & 0.533 & 0.248 & 0.8 & 0.179 & 0.6 & 0.219 \\
\hline Spindle cell sarcomas & 4 & 0.5 & 0.25 & 0.5 & 0.25 & 0.5 & 0.25 & . & . \\
\hline Leiomyosarcoma & 4 & 0.5 & 0.25 & $\cdot$ & . & 0.5 & 0.25 & $\cdot$ & . \\
\hline Myofibrosarcoma & 3 & 0.667 & 0.272 & . & . & 0.33 & 0.272 & . & . \\
\hline Synovial sarcoma & 3 & 1.0 & 0.0 & 1.0 & 0.0 & 0.5 & 0.354 & 0.5 & 0.354 \\
\hline
\end{tabular}


Figure 1.

a.
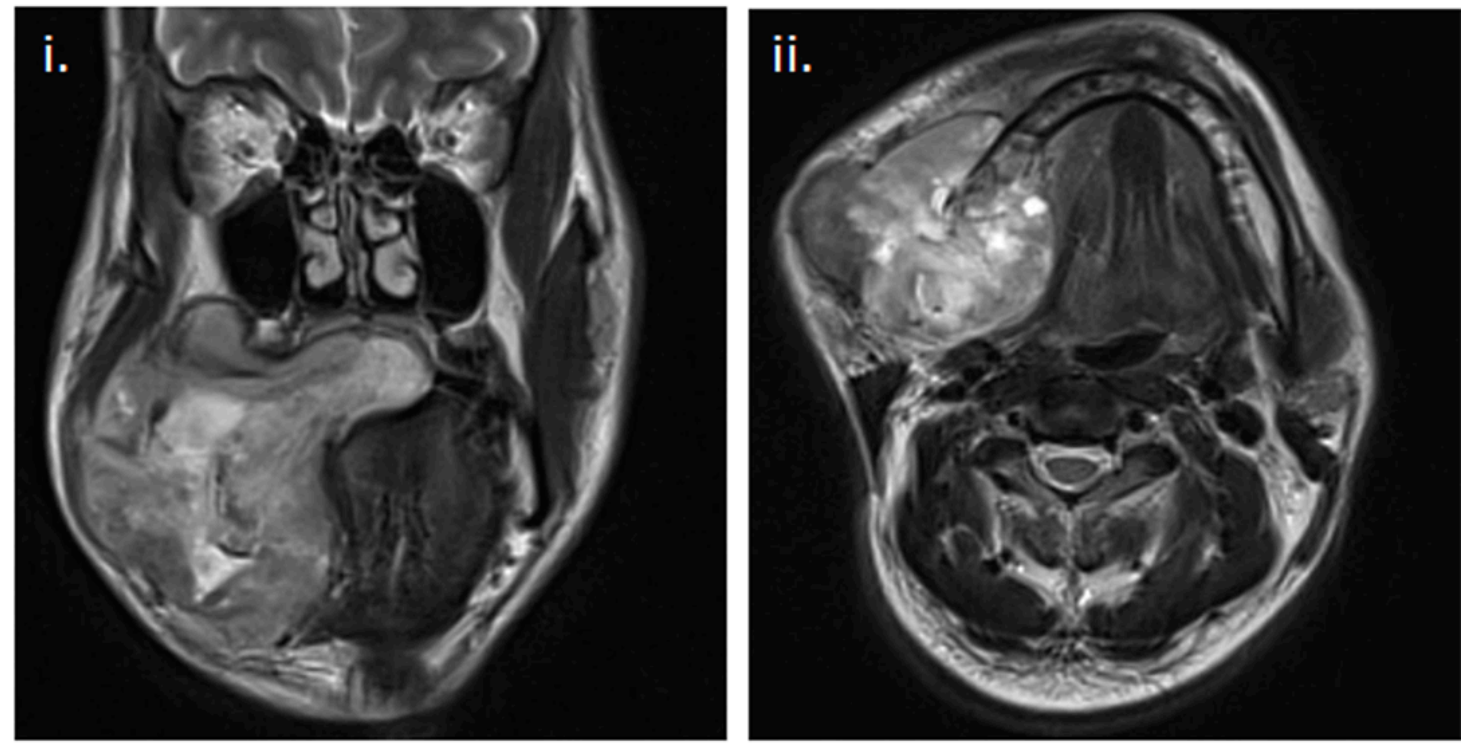

b.
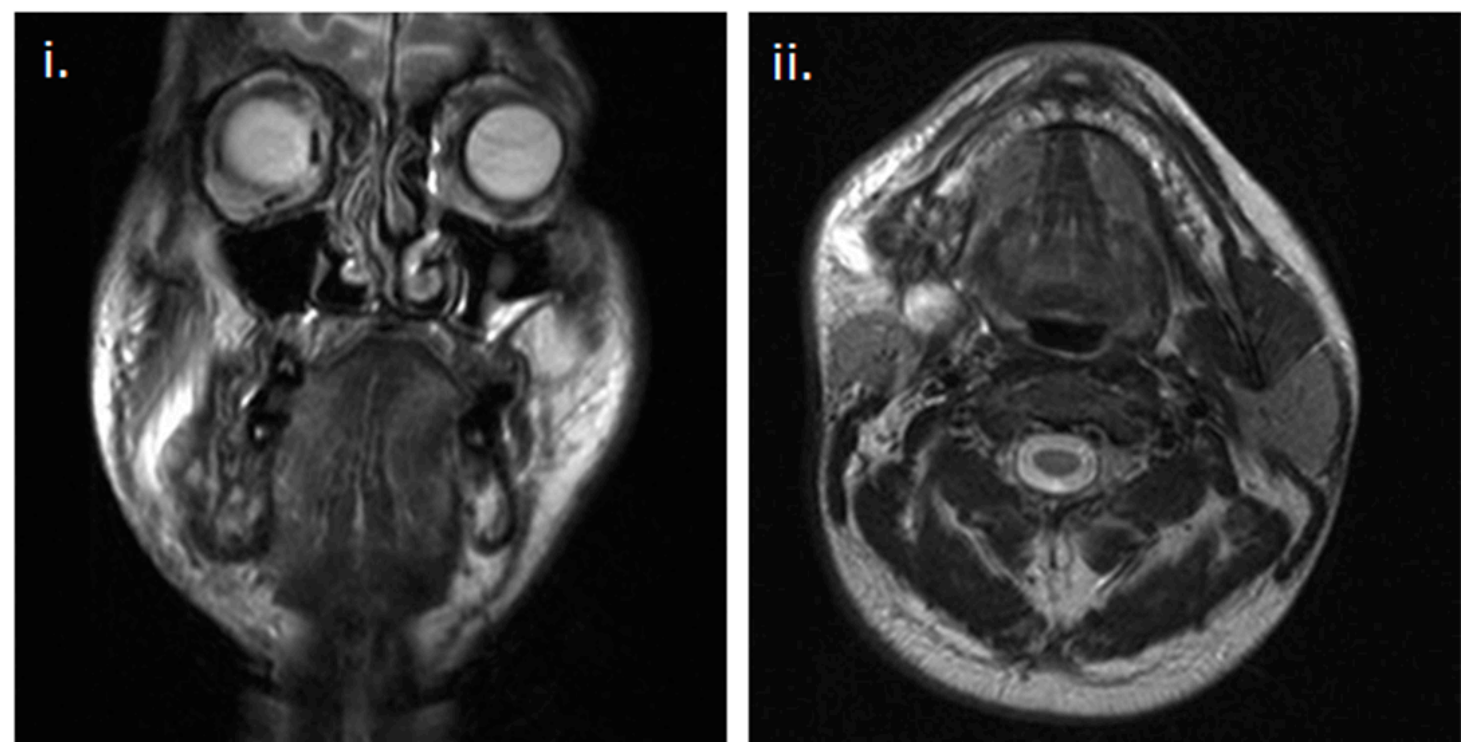
Figure 2.

a.

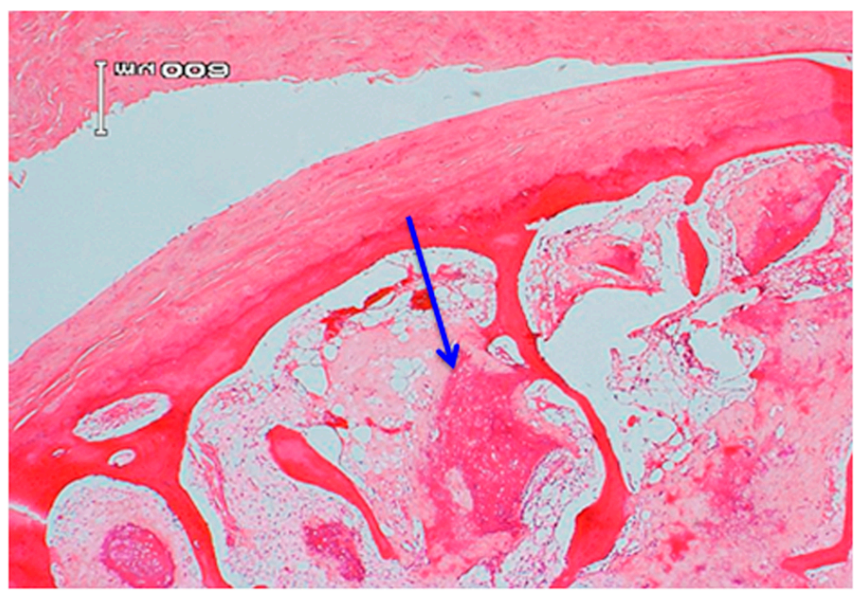

b.
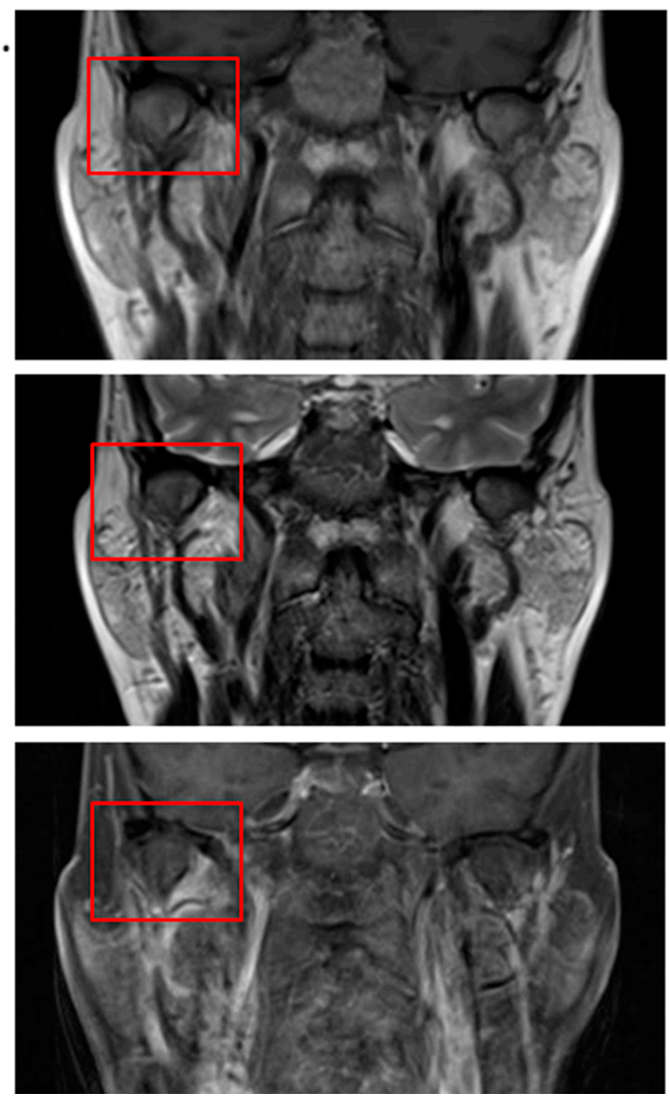
Figure 3.

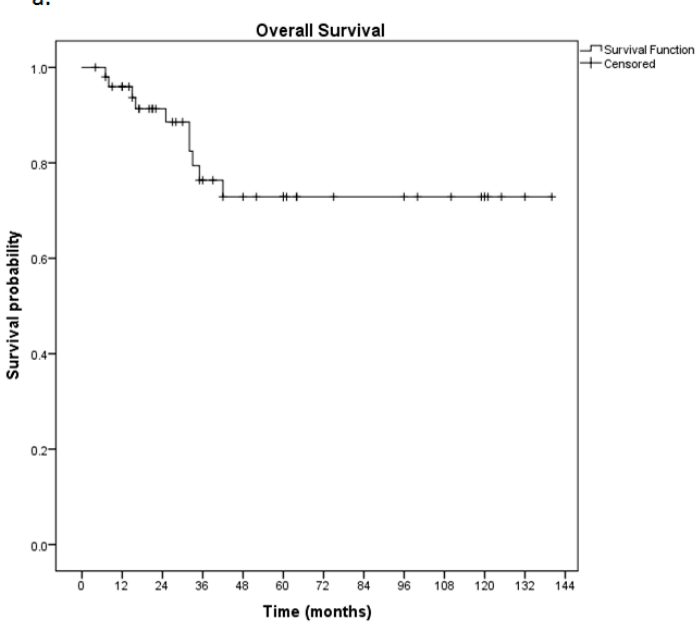

b.

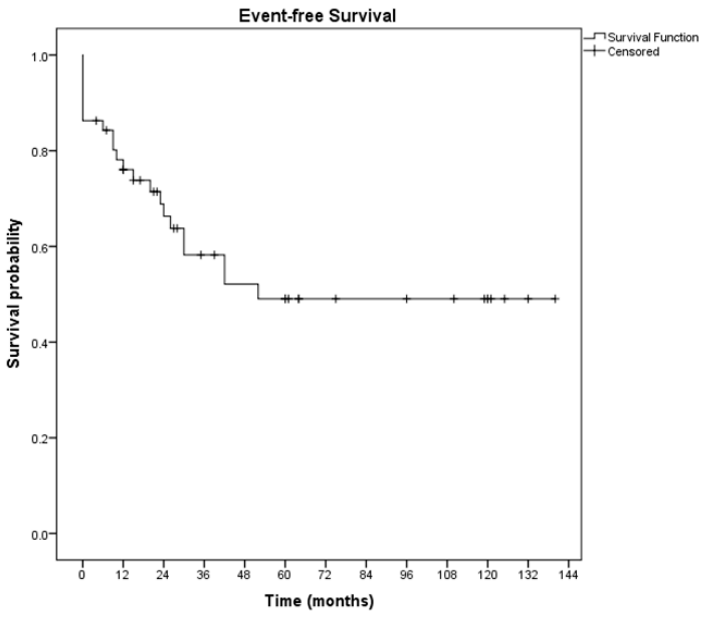


Figure 4.
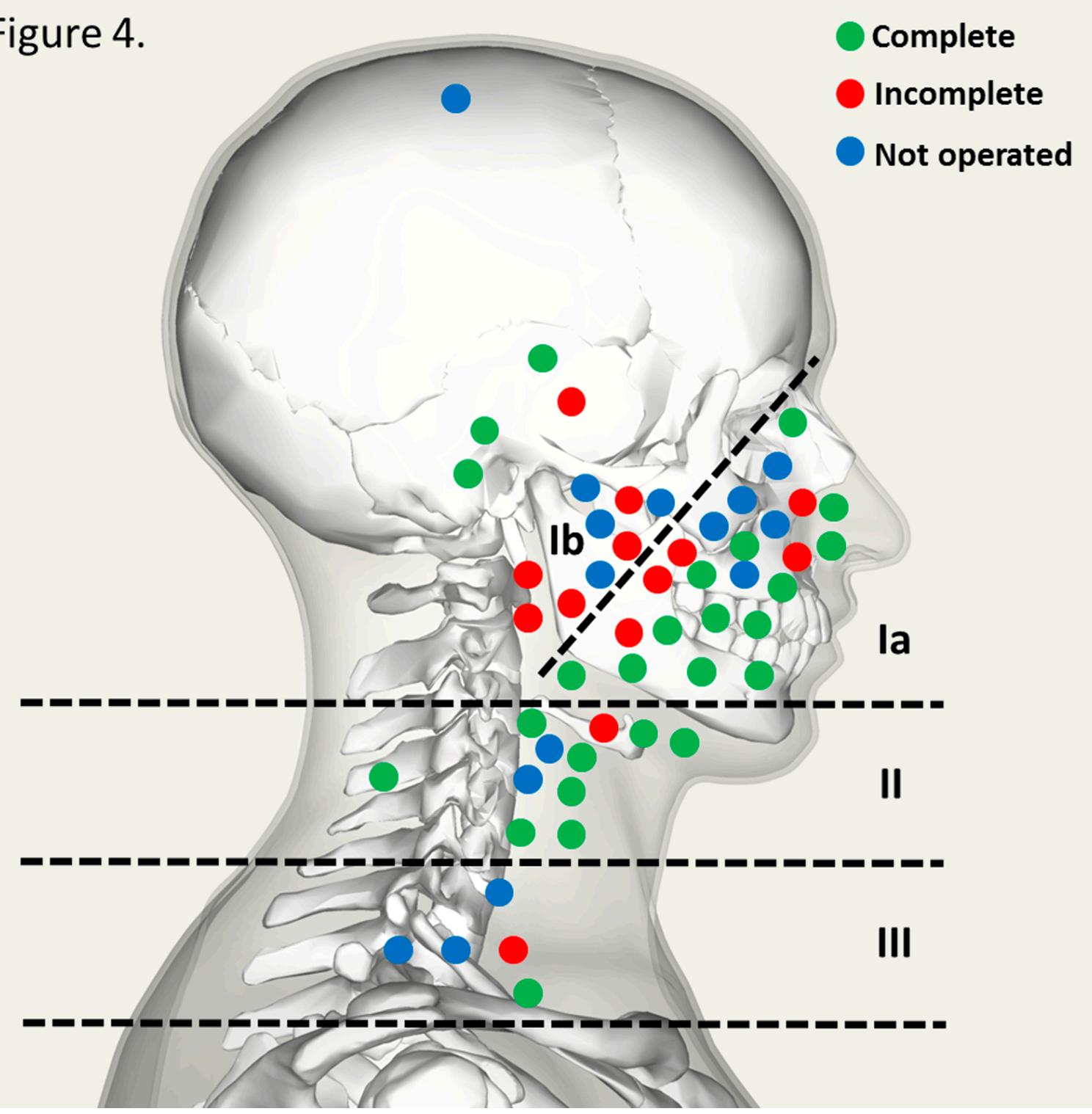

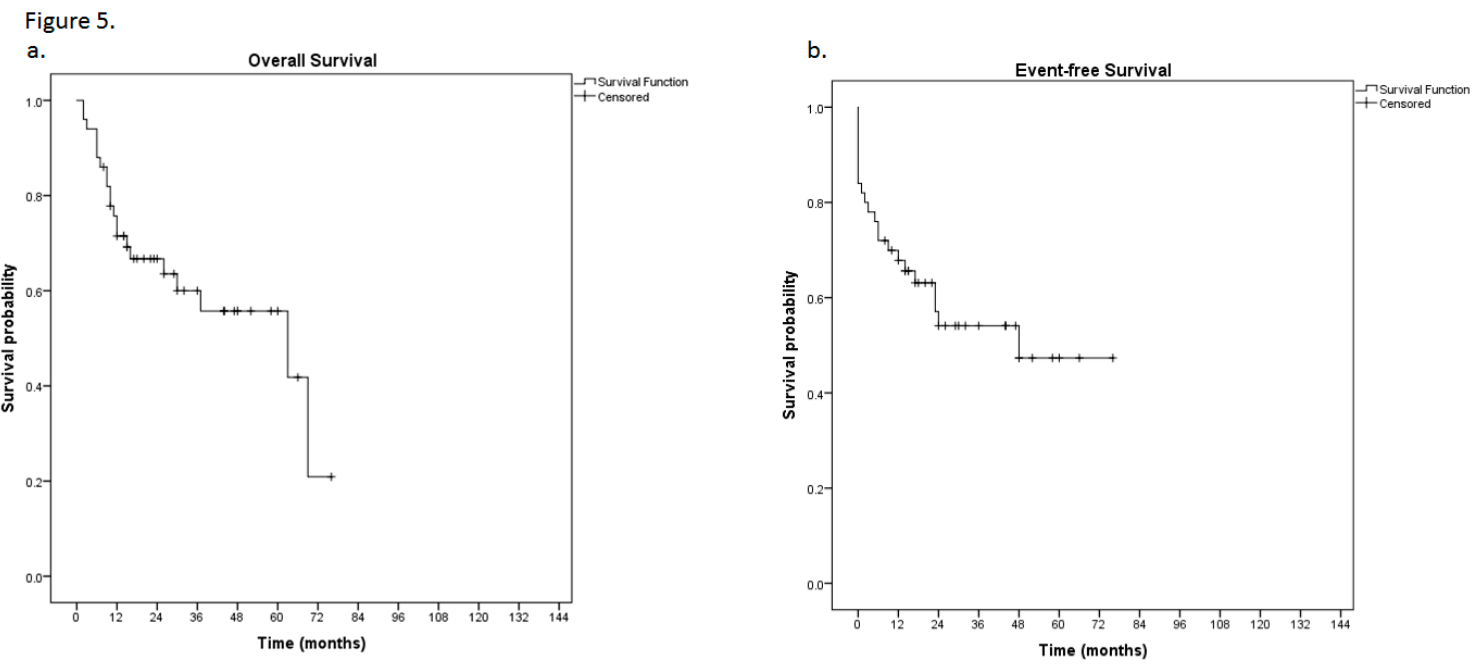
Figure 6.

a.

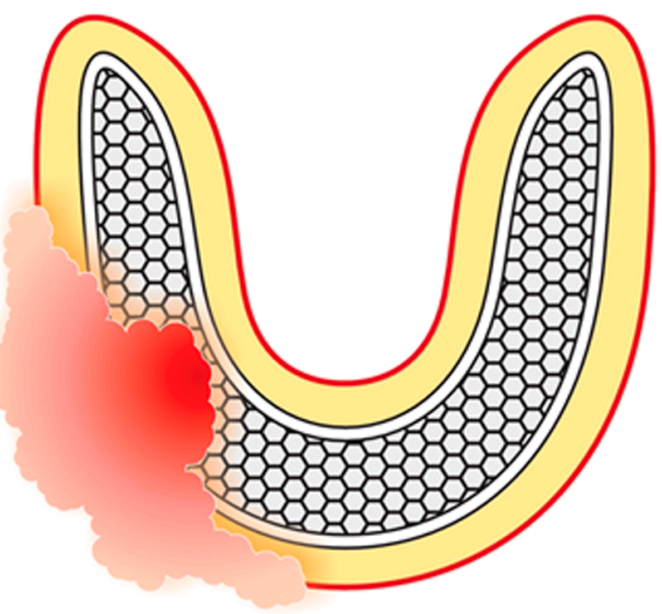

b.

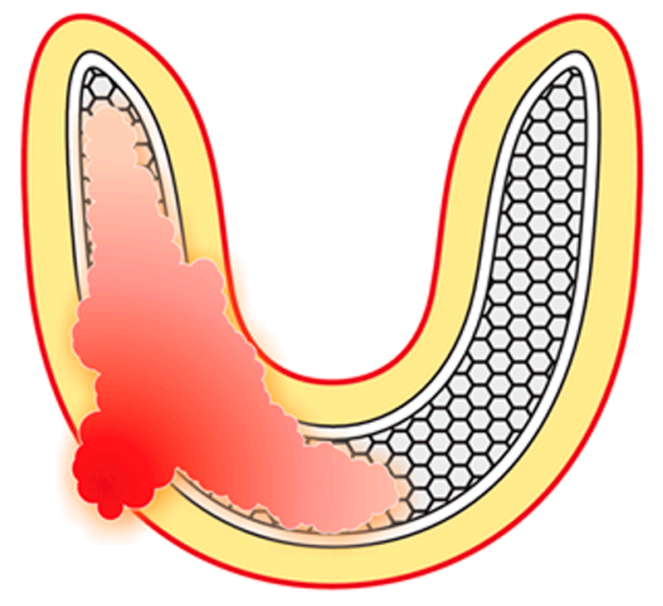

